\title{
Synthesis of Oscillators Using Limit Variables and NAM Expansion
}

\begin{abstract}
Ahmed M. Soliman
Electronics and Communication Engineering Department, Faculty of Engineering, Cairo University, Giza 12613, Egypt

Correspondence should be addressed to Ahmed M. Soliman, asoliman@ieee.org

Received 12 January 2011; Accepted 7 March 2011

Academic Editor: Jiun Wei Horng

Copyright ( 2011 Ahmed M. Soliman. This is an open access article distributed under the Creative Commons Attribution License, which permits unrestricted use, distribution, and reproduction in any medium, provided the original work is properly cited.

A systematic synthesis procedure for generating second-order grounded passive element canonic oscillators is given. The synthesis procedure is based on using nodal admittance matrix (NAM) expansion with the bracket method as well as using the infinity parameters. The resulting derived oscillators include circuits using various types of current conveyors. Two classes of oscillators are considered in this paper, and they have the advantages of having independent control on the condition of oscillation and on the frequency of oscillation by varying two different grounded resistors. The two classes of oscillators considered can be easily compensated for the parasitic element effects introduced by the current conveyors. This paper is considered to be continuation to the recently published paper on oscillators using NAM expansion D. G. Haigh et al. (2006). This is the first paper in the literature which uses limit-variables called infinity-variables D. G. Haigh et al. (2005) in the synthesis of oscillator circuits. Simulation results demonstrating the practicality of some of the generated circuits are included.
\end{abstract}

\section{Introduction}

The symbolic framework for systematic synthesis of linear active circuits based on NAM expansion was introduced and presented in [1-4]. The NAM expansion was limited to the use of nullators and norators as the two pathological elements [5].

For the nullator shown in Figure $1(\mathrm{a}), V=I=0$. The norator shown in Figure 1(b) imposes no constraints on its voltage and current. Additional pathological elements called mirror elements were introduced in [6-8] to describe the voltage and current reversing actions.

The voltage mirror (VM) shown in Figure $1(\mathrm{c})$ is a lossless two-port circuit element used to represent an ideal voltage reversing action, and it is described by

$$
\begin{gathered}
V_{1}=-V_{2}, \\
I_{1}=I_{2}=0 .
\end{gathered}
$$

The current mirror (CM) shown in Figure 1(d) is a two-port circuit element used to represent an ideal current reversing action, and it is described by:

$$
\begin{gathered}
V_{1} \text { and } V_{2} \text { are arbitrary, } \\
I_{1}=I_{2} \text {, and they are also arbitrary. }
\end{gathered}
$$

Recently, the systematic synthesis method based on NAM expansion using nullor elements has been extended to accommodate mirror elements. This results in a generalized framework encompassing all pathological elements for ideal description of active elements [9-11]. Accordingly, more alternative realizations are possible and a wide range of active devices can be used in the synthesis.

In this paper, the conventional systematic synthesis framework using NAM expansion is used to synthesize grounded passive element oscillator circuits. The active building blocks that are considered are the current conveyors (CCII) [12], inverting current conveyors (ICCII) [6], balanced output CCII (BOCCII), double output CCII (DOCCII), balanced output ICCII (BOICCII), double output ICCII (DOICCII), and the differential voltage current conveyor (DVCC) [13] also known as the differential difference current conveyor (DDCC) [14].

\section{Formulation of the NAM Equation}

The oscillators considered in this paper are grounded resistors and grounded capacitors second-order canonic (using two capacitors) oscillators having independent control on the condition of oscillation and on the frequency of oscillation by varying two different resistors. 
The state equations are described by the following matrix equation:

$$
\left[\begin{array}{l}
\frac{d v_{1}}{d t} \\
\frac{d v_{2}}{d t}
\end{array}\right]=\left[\begin{array}{ll}
a_{11} & a_{12} \\
a_{21} & a_{22}
\end{array}\right]\left[\begin{array}{l}
v_{1} \\
v_{2}
\end{array}\right]
$$

The condition of oscillation and the radian frequency of oscillation are given by [15]

$$
\begin{gathered}
a_{11}+a_{22}=0, \\
\omega_{\mathrm{o}}=\sqrt{a_{11} a_{22}-a_{12} a_{21}} .
\end{gathered}
$$

If both $a_{11}$ and $a_{22}$ are zero, there will be no control on the condition of oscillation. For simplicity, it is assumed that either $a_{22}$ (or $a_{11}$ ) is zero so that the radian frequency of oscillation is controlled only by $a_{12}$ and $a_{21}$, and they must have opposite signs. In this case, the condition of oscillation is $a_{11}=0$ (or $a_{22}=0$ ).

Two classes of oscillator circuits are considered in this paper. The class I oscillator is a five-node oscillator using four resistors one of them shares a node with one of the capacitors as shown in Figure 2(a). The class II oscillator is a four-node oscillator using three grounded resistors, one of them shares a node with one of the capacitors as shown in Figure 2(b).

\section{Class I Oscillators}

The generalized class I oscillator configuration can be described by the following state equation:

$$
\left[\begin{array}{c}
C_{1} \frac{d v_{1}}{d t} \\
C_{2} \frac{d v_{2}}{d t}
\end{array}\right]=\left[\begin{array}{cc}
G_{4}-G_{1} & \mp G_{3} \\
\pm G_{2} & 0
\end{array}\right]\left[\begin{array}{l}
v_{1} \\
v_{2}
\end{array}\right] .
$$

The admittance matrix $Y$ of the two-port oscillator circuit taking the capacitors $C_{1}$ and $C_{2}$ as external elements at ports 1 and 2 is formulated from the above equation by interchanging the signs of the admittance parameters. There are two types that belong to class I oscillators.

The type-A for which the NAM is given by

$$
Y=\left[\begin{array}{cc}
G_{1}-G_{4} & G_{3} \\
-G_{2} & 0
\end{array}\right],
$$

and the NAM for type-B is given by

$$
Y=\left[\begin{array}{cc}
G_{1}-G_{4} & -G_{3} \\
G_{2} & 0
\end{array}\right] .
$$

Table 1 includes the admittance matrix $Y$ of the two types of class I. To limit the paper length only type A is considered in this paper. The above NAM equations can be expanded in several alternative ways resulting in different oscillator circuits as described next.
It should be noted that after the synthesis procedure is completed the pathological elements are paired to realize the proper CCII- [12] or ICCII- [6] based oscillator circuit as follows:

The nullator, and norator with a common terminal realizes a CCII-, the nullator and CM with a common terminal realizes a CCII+, the VM, and norator with a common terminal realizes an ICCII-, the VM, and CM with a common terminal realizes an ICCII+.

3.1. Realization I. The expansion of the matrix $Y$ is demonstrated in several steps as follows. Starting from (5a), adding two blank rows and columns, and connecting a nullator between node 2 and node 4 to move $G_{3}$ to position 1,4 as follows:

$$
Y=\left[\begin{array}{cccc}
G_{1}-G_{4} & \stackrel{0}{0} & 0 & G_{3} \\
-G_{2} & 0 & 0 & 0 \\
0 & 0 & 0 & 0 \\
0 & 0 & 0 & 0
\end{array}\right]
$$

Next a norator from node 1 to node 4 is introduced to move $G_{3}$ to the diagonal position 4, 4 as follows:

$$
Y=\left[\begin{array}{cccc}
G_{1}-G_{4} & 0 & 0 & 0 \\
-G_{2} & 0 & 0 & 0 \\
0 & 0 & 0 & 0 \\
0 & 0 & 0 & G_{3}
\end{array}\right]
$$

A second nullator from node 1 to node 3 is introduced to move $-G_{2}$ to positions 2,3 as follows:

$$
\left.Y=\left[\begin{array}{cccc}
G_{1}-G_{4} & 0 & 0 & 0 \\
0 & 0 & -G_{2} & 0 \\
0 & 0 & 0 & 0 \\
0 & 0 & 0 & G_{3}
\end{array}\right]\right]
$$

Next, a CM is connected between node 2 and node 3 to move $-G_{2}$ to become $G_{2}$ at the diagonal positions 3, 3 as follows:

$$
\left.Y=\left[\begin{array}{cccc}
G_{1}-G_{4} & 0 & 0 & 0 \\
0 & 0 & 0 & 0 \\
0 & 0 & G_{2} & 0 \\
0 & 0 & 0 & G_{3}
\end{array}\right]\right\} .
$$

Adding an infinity term to the position 1, 1 and subtracting an equivalent term as demonstrated in [1], it follows that

$$
\left.Y=\left[\begin{array}{cccc}
G_{1}-G_{4}+\infty_{1}-\frac{\infty_{1}^{2}}{\infty_{1}} & \multicolumn{0}{c}{0} & 0 \\
0 & 0 & 0 & 0 \\
0 & 0 & G_{2} & 0 \\
0 & 0 & 0 & G_{3}
\end{array}\right]\right] .
$$


TABLE 1: A summary of the parameters of the two classes of oscillators.

\begin{tabular}{lccccc}
\hline Class & Type-A NAM & Type-B NAM & Oscillation Condition & $\omega_{\mathrm{O}}$ & Adjoint class \\
\hline I & {$\left[\begin{array}{ccc}G_{1}-G_{4} \\
-G_{2} & G_{3}\end{array}\right]$} & {$\left[\begin{array}{ccc}G_{1}-G_{4} & -G_{3} \\
G_{2} & 0\end{array}\right]$} & $G_{4}=G_{1}$ & $\omega_{\mathrm{O}}=\sqrt{G_{2} G_{3} / C_{1} C_{2}}$ & I-Ad \\
I-Ad & {$\left[\begin{array}{ccc}G_{1}-G_{4} & -G_{2} \\
G_{3} & 0\end{array}\right]$} & {$\left[\begin{array}{ccc}G_{1}-G_{4} \\
-G_{3} & 0\end{array}\right]$} & $G_{4}=G_{1}$ & $\omega_{\mathrm{O}}=\sqrt{G_{2} G_{3} / C_{1} C_{2}}$ & I \\
II & {$\left[\begin{array}{ccc}G_{1}-G_{2} & -G_{3} \\
G_{2} & 0\end{array}\right]$} & {$\left[\begin{array}{ccc}G_{1}-G_{2} & G_{3} \\
-G_{2} & 0\end{array}\right]$} & $G_{2}=G_{1}$ & $\omega_{\mathrm{O}}=\sqrt{G_{2} G_{3} / C_{1} C_{2}}$ & II-Ad \\
II-Ad & {$\left[\begin{array}{ccc}G_{1}-G_{2} & G_{2} \\
-G_{3} & 0\end{array}\right]$} & {$\left[\begin{array}{cc}G_{1}-G_{2} & -G_{2} \\
G_{3} & 0\end{array}\right]$} & $G_{2}=G_{1}$ & $\omega_{\mathrm{O}}=\sqrt{G_{2} G_{3} / C_{1} C_{2}}$ & II \\
\hline
\end{tabular}

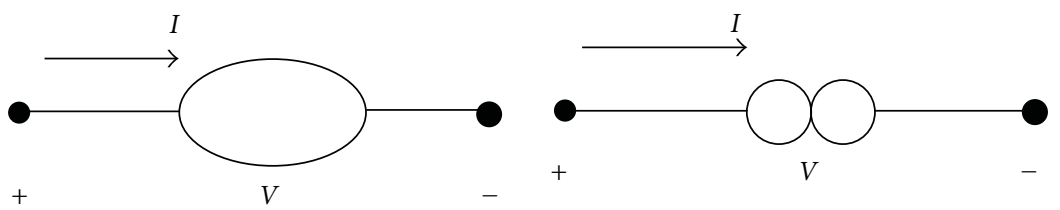

(a)

(b)



(c)

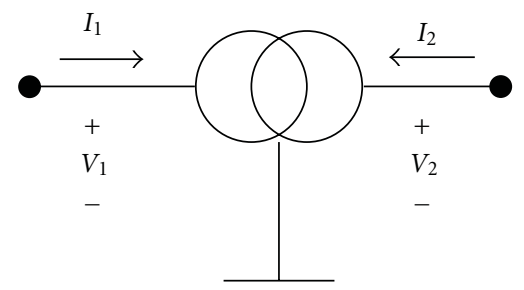

(d)

FIgURE 1: The pathological elements: (a) nullator, (b) norator, (c) voltage mirror, (d) current mirror.

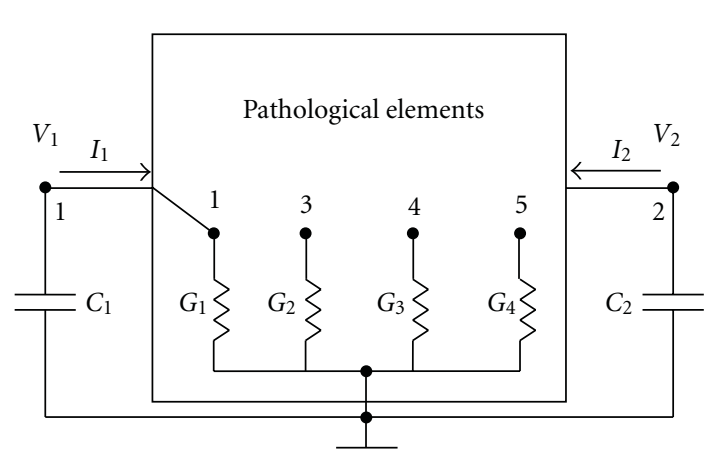

(a)

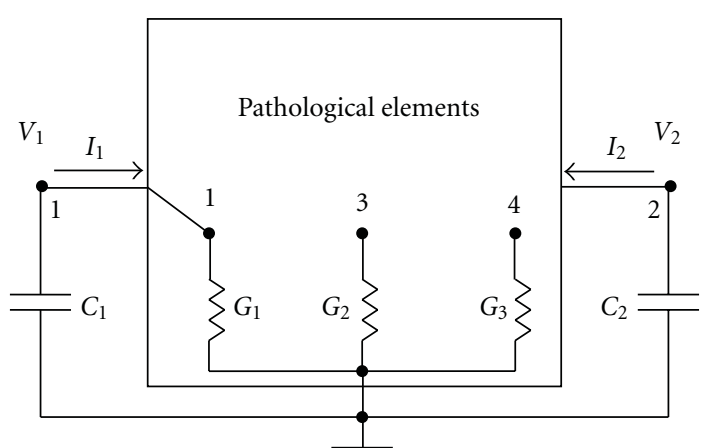

(b)

FIgURE 2: (a) Class-I four grounded resistor five node oscillator. (b) Class-II three grounded resistor four node oscillator.

Adding a fifth blank row and column and applying pivotal expansion [2] to the fourth term in the 1,1 position it follows that
The infinity parameters added will move $-G_{4}$ to become $G_{4}$ at the diagonal positions 5, 5 as follows:






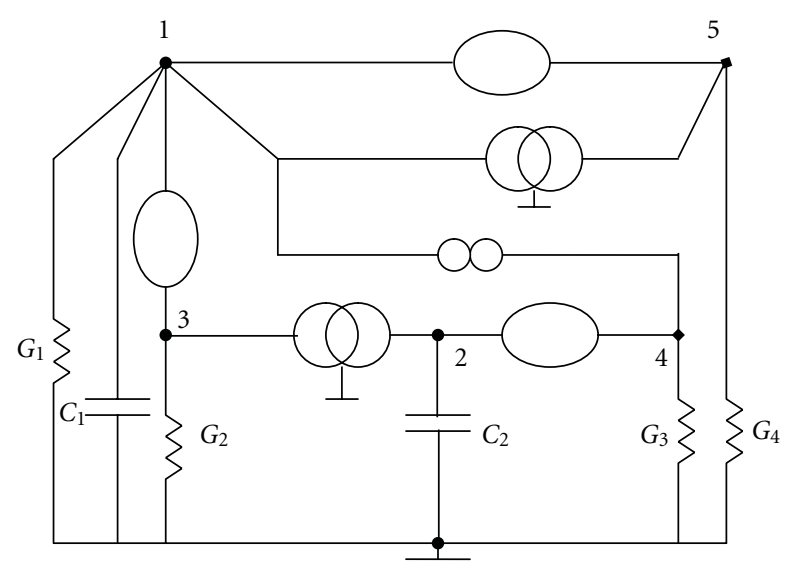

(a)

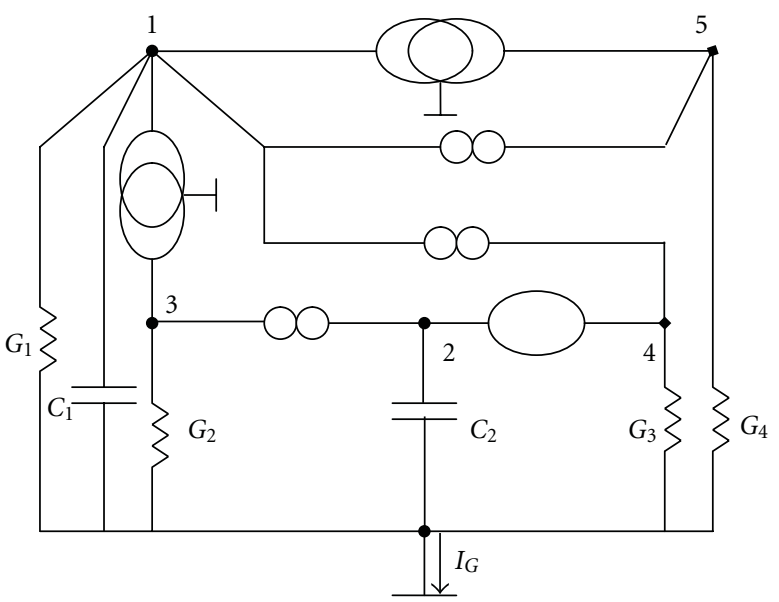

(b)



(c)

Figure 3: (a) Realization I of Class-I oscillator. (b) Realization II of Class-I oscillator, (c) Realization III of Class-I oscillator.

Figure 3(a) represents the pathological element realization of the above equation after connecting the two capacitors at nodes 1 and 2.

3.2. Realization II. A new circuit that can be generated from (5a) by alternative matrix expansion is given next.
Starting from the $Y$ matrix given by (7), A VM is connected between nodes 1 and 3 to move $-G_{2}$ to become $G_{2}$ at the position 2, 3 as follows:

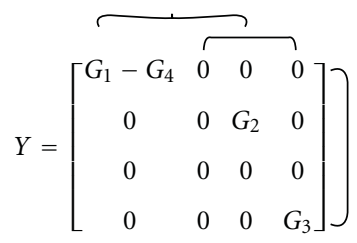

A norator is added next between nodes 2 and 3 to move $G_{2}$ to the diagonal position 3, 3 as follows:

$$
\left.Y=\left[\begin{array}{cccc}
G_{1}-G_{4} & 0 & 0 & 0 \\
0 & 0 & 0 & 0 \\
0 & 0 & G_{2} & 0 \\
0 & 0 & 0 & G_{3}
\end{array}\right]\right]
$$

Adding a fifth blank row and column, adding infinity parameter element to the 1,1 position, subtracting an equivalent term, and apply pivotal expansion in alternative way from (11), it follows that

$$
\left.Y=\left[\begin{array}{ccccc}
G_{1}-G_{4}+\infty_{1} & 0 & 0 & 0 & \infty_{1} \\
0 & 0 & 0 & 0 & 0 \\
0 & 0 & G_{2} & 0 & 0 \\
0 & 0 & 0 & G_{3} & 0 \\
-\infty_{1} & 0 & 0 & 0 & -\infty_{1}
\end{array}\right]\right] .
$$

The infinity parameters added will move $-G_{4}$ to become $G_{4}$ at the diagonal positions 5,5 as follows:

$$
\left.Y=\left[\begin{array}{ccccc}
G_{1}+\infty_{1} & 0 & 0 & 0 & \infty_{1} \\
0 & 0 & 0 & 0 & 0 \\
0 & 0 & G_{2} & 0 & 0 \\
0 & 0 & 0 & G_{3} & 0 \\
-\infty_{1} & 0 & 0 & 0 & G_{4}-\infty_{1}
\end{array}\right]\right]
$$

Figure 3(b) represents the realization of (16) after connecting the two capacitors at nodes 1 and 2, which is realizable using two ICCII- and one CCII-.

3.3. Realization III. Another new circuit that can be generated from (5a) by alternative matrix expansion is given next.

Starting from the $Y$ matrix given by (7) and by successive NAM expansion steps to move $-G_{2}$ and $G_{3}$ to the diagonal positions 3,3 and 4,4 , respectively, then using infinity 




(a)



(b)

FIgUre 4: (a) Realization of Figure 3(a) using two CCII+ and one CCII- [16]. (b) Realization of Figure 3(b) using two ICCII- and one CCII-.

parameters to move $-G_{4}$ to the diagonal position 5,5 , the following NAM is obtained:

$$
\left.Y=\left[\begin{array}{ccccc}
G_{1}+\infty_{1} & 0 & 0 & 0 & -\infty_{1} \\
0 & 0 & 0 & 0 & 0 \\
0 & 0 & G_{2} & 0 & 0 \\
0 & 0 & 0 & G_{3} & 0 \\
\infty_{1} & 0 & 0 & 0 & G_{4}-\infty_{1}
\end{array}\right]\right]
$$

Figure 3(c) represents the realization of the above equation after connecting the two capacitors at nodes 1 and 2 . The circuit is realizable using one ICCII-, one CCII+ and one CCII-.

Figure 4(a) represents a two CCII+ one CCII- circuit realizing Figure 3(a) [16]. Figure 4(b) represents a two ICCII- one CCII- circuit realizing Figure 3(b). This circuit represents a new oscillator with flotation property, that is $I_{G}=0$.
There are a total of sixteen circuits having the same circuit topology as that of Figure 4(a). Eight circuits belong to type-A, and are generated from (5a) and the other eight circuits belong to type-B and are generated from ( $5 b)$.

The topology of the class I oscillators generated in this paper has the advantage that the parasitic resistance $R_{X 1}$ can be easily compensated by subtracting its value from the design value of $R_{2}$, the parasitic resistance $R_{X 2}$ can be easily compensated by subtracting its value from the design value of $R_{3}$, and the parasitic resistance $R_{X 3}$ can be easily compensated by subtracting its value from the design value of $R_{4}$. Similarly, the parasitic capacitance $C_{Z 1}$ can be easily compensated by subtracting its value from the design value of $C_{2}$. Similarly, the parasitic capacitances $\left(C_{Z 2}+C_{Z 3}\right)$ can be easily compensated by subtracting their sum value from the design value of $C_{1}$.

\section{Adjoint of Class I Type-A Oscillators}

The two classes of oscillators considered in this paper can lead to additional oscillator families based on the adjoint transformation $[18,19]$.

The NAM equations of the two adjoint classes are given in Table 1, and they are the transposition of the original NAM equations.

To limit paper length only realization I of class I typeA defined as class I-Ad where Ad stands for adjoint will be considered.

The NAM equation for the class I-Ad oscillator is given by

$$
Y=\left[\begin{array}{cc}
G_{1}-G_{4} & -G_{2} \\
G_{3} & 0
\end{array}\right] .
$$

First two blank rows and columns are added, and a nullator is connected between nodes 1 and 4 in order to move $G_{3}$ to position 2, 4 as follows:

$$
Y=\left[\begin{array}{cccc}
G_{1}-G_{4} & -G_{2} & 0 & 0 \\
0 & 0 & 0 & G_{3} \\
0 & 0 & 0 & 0 \\
0 & 0 & 0 & 0
\end{array}\right]
$$

Next, a norator is connected between nodes 2 and 4 to move $G_{3}$ to the diagonal position 4,4 as follows:

$$
\left.Y=\left[\begin{array}{cccc}
G_{1}-G_{4} & -G_{2} & 0 & 0 \\
0 & 0 & 0 & 0 \\
0 & 0 & 0 & 0 \\
0 & 0 & 0 & G_{3}
\end{array}\right]\right] .
$$


Next, a VM is connected between nodes 2 and 3 to move $-G_{2}$ to become $G_{2}$ at the position 1, 3 as follows:

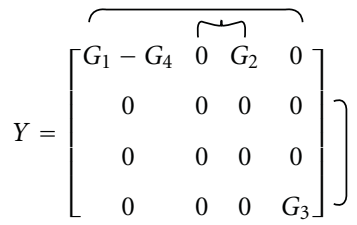

Next, a norator is connected between node 1 and node 3 to move $G_{2}$ to the diagonal position 3, 3 as follows:

$$
\left.Y=\left[\begin{array}{cccc}
G_{1}-G_{4} & 0 & 0 & 0 \\
0 & 0 & 0 & 0 \\
0 & 0 & G_{2} & 0 \\
0 & 0 & 0 & G_{3}
\end{array}\right]\right]
$$

Adding a fifth blank row and column and using infinity parameters to move $-G_{4}$ to the diagonal position 5,5 the following NAM is obtained:

$$
\left.Y=\left[\begin{array}{ccccc}
G_{1}+\infty_{1} & 0 & 0 & 0 & \infty_{1} \\
0 & 0 & 0 & 0 & 0 \\
0 & 0 & G_{2} & 0 & 0 \\
0 & 0 & 0 & G_{3} & 0 \\
-\infty_{1} & 0 & 0 & 0 & G_{4}-\infty_{1}
\end{array}\right]\right] .
$$

Figure 5(a) represents the pathological realization of the above equation after connecting the capacitors $C_{1}$ and $C_{2}$ at nodes 1 and 2, respectively.

Figure 5(b) represents the circuit realization using two ICCII- and one CCII-. This is the adjoint circuit to that of Figure 4(a), noting that the adjoint of CCII+ number 1 in Figure 4(a) is the ICCII- number 2 in Figure 5(b), the adjoint of CCII- number 2 in Figure $4(a)$ is the CCIInumber 1 in Figure 5(b), and the adjoint of CCII+ number 3 in Figure 4(a) is the ICCII- number 3 in Figure 5(b). It should also be noted that the circuit of Figure $5(\mathrm{~b})$ has a similar topology to the circuit of Figure 4(b) except for the interchange of the two branches of $G_{2}$ and $G_{3}$. It is seen that the topology of the class I oscillators generated in this paper is self-adjoint, that is, eight of the generated circuits are the adjoints of the other eight circuits.

\section{Class II Oscillators}

The two types of class II oscillators are considered next. Generation method of this class of oscillators was given recently in [20] based on using three single output CCII and ICCII, two grounded capacitors, and four grounded resistors two of them are equal. Then, the two CCII having equal resistors connected to their port $X$ are combined to realize a BOCCII or a DOCCII depending on the $Z$ port polarity of



(a)

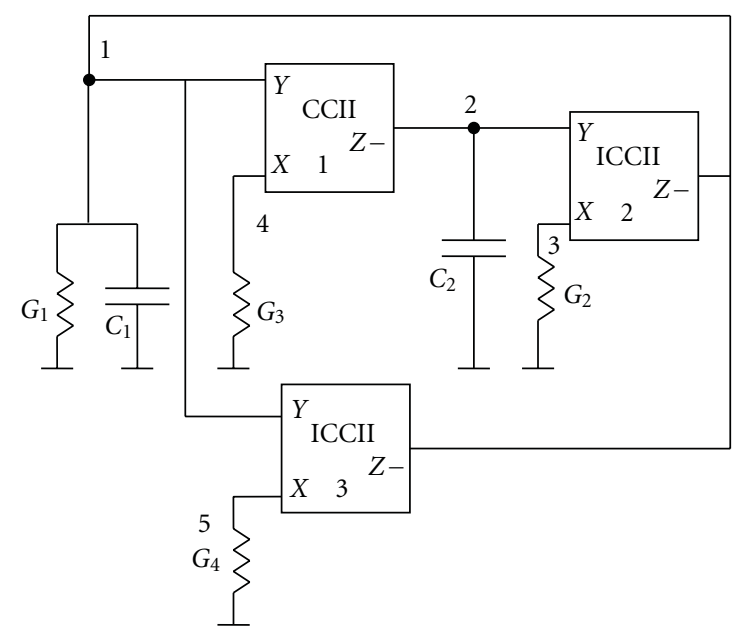

(b)

Figure 5: (a) Realization I-of Class I-A oscillator. (b)Realization of Figure 5(a) using two ICCII- and one CCII-.

the two CCII. An alternative and much simpler approach in the generation of this class of oscillators without expanding the NAM equation to a $5 \times 5$ matrix equation (five-node circuit) is given next.

It should be noted that in the bracket method, the brackets representing a nullator, norator, and a CM with a common node realize a BOCCII. The brackets representing a nullator, two CM with a common node realize a DOCCII++. The brackets representing a nullator, two norator with a common node realize a DOCCII-.

The brackets representing a VM, norator, and a CM with a common node realize a BOICCII. The brackets representing a $\mathrm{VM}$, two $\mathrm{CM}$ with a common node realize a DOICCII++. The brackets representing a VM, two norator with a common node realize a DOICCII-.

5.1. Class II Type-A. The nodal admittance matrix for the four node class II type-A oscillator is given by

$$
Y=\left[\begin{array}{cc}
G_{1}-G_{2} & -G_{3} \\
G_{2} & 0
\end{array}\right] .
$$




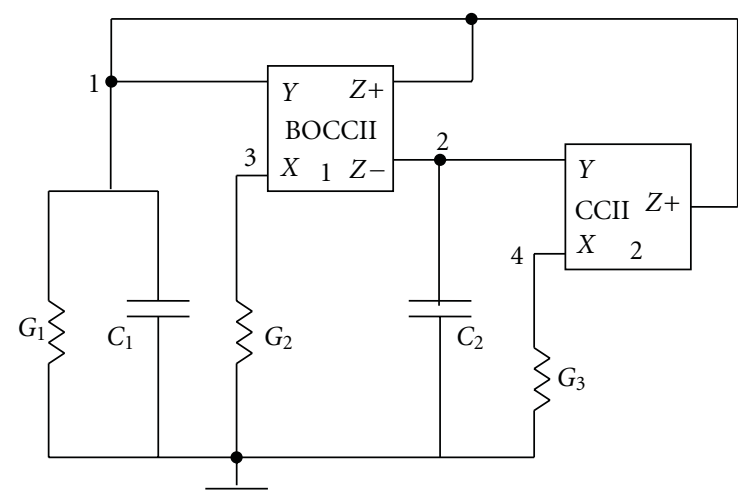

FIgURE 6: Realization I of class II type-A oscillator [17].

5.1.1. Realization I. The NAM expansion starts by adding two blank rows and columns and using a nullator to link nodes 2 and 4 and a CM to link nodes 1 and 4 in order to move $-G_{3}$ to become $G_{3}$ at the diagonal position 4,4 as follows:

$$
\left.Y=\left[\begin{array}{cccc}
G_{1}-G_{2} & 0 & 0 & 0 \\
G_{2} & 0 & 0 & 0 \\
0 & 0 & 0 & 0 \\
0 & 0 & 0 & G_{3}
\end{array}\right]\right] .
$$

A nullator is added between nodes 1 and 3 to move $-G_{2}$ and $G_{2}$ to positions 1, 3 and 2, 3, respectively as follows:

$$
\left.Y=\left[\begin{array}{cccc}
G_{1} & 0 & -G_{2} & 0 \\
0 & 0 & G_{2} & 0 \\
0 & 0 & 0 & 0 \\
0 & 0 & 0 & G_{3}
\end{array}\right]\right] .
$$

A CM is added between nodes 1 and 3 to move $-G_{2}$ to the diagonal position 3, 3 and a norator is added between nodes 2 and 3 to move $G_{2}$ to the diagonal position 3, 3 as follows:

$$
\left.\left.Y=\left[\begin{array}{cccc}
G_{1} & 0 & 0 & 0 \\
0 & 0 & 0 & 0 \\
0 & 0 & G_{2} & 0 \\
0 & 0 & 0 & G_{3}
\end{array}\right]\right]\right\} .
$$

The above equation is obtained after applying the scaling of row rules demonstrated in [2] to row three and with a scaling factor of one half.

It should be noted the brackets representing the nullator between nodes 1, 3, the norator between nodes 3, 2 and the $\mathrm{CM}$ between nodes 3, 1 realize a BOCCII.

The nullator between nodes 2, 4 and the CM between nodes 4, 1 realize CCII+. Figure 6 realizes the above equation using a BOCCII and a CCII+ [17].
Three more circuits that belong to class II type-A can be generated in a similar way and are not included to limit paper length.

5.2. Class II-Type B. The NAM equation for the class II type$\mathrm{B}$ oscillator is given by

$$
Y=\left[\begin{array}{cc}
G_{1}-G_{2} & G_{3} \\
-G_{2} & 0
\end{array}\right]
$$

5.2.1. Realization I. Starting from (28) and adding two blank rows and columns then following successive NAM expansion steps the following NAM is obtained:

$$
\left.\left.Y=\left[\begin{array}{cccc}
G_{1} & 0 & 0 & 0 \\
0 & 0 & 0 & 0 \\
0 & 0 & G_{2} & 0 \\
0 & 0 & 0 & G_{3}
\end{array}\right]\right]\right] .
$$

It should be noted that the brackets representing the nullator joining nodes 1, 3, the CM joining nodes 3, 2 and the CM joining nodes 3, 1 realize a DOCCII++. The nullator between nodes 2, 4 and the norator between nodes 4 , 1 realize CCIIas shown in Figure 7(a).

5.2.2. Realization II. Following successive NAM expansion steps starting from (28) after adding two blank rows and columns, the following NAM equation is obtained:

$$
\left.\left.Y=\left[\begin{array}{cccc}
G_{1} & 0 & 0 & 0 \\
0 & 0 & 0 & 0 \\
0 & 0 & G_{2} & 0 \\
0 & 0 & 0 & G_{3}
\end{array}\right]\right]\right] .
$$

Figure $7(\mathrm{~b})$ realizes the above equation using a DOCCII++ and an ICCII+.

5.2.3. Realization III. Starting from (28) and adding two blank rows and columns then following successive NAM expansion steps, the following $Y$ matrix is obtained:

$$
\left.\left.Y=\left[\begin{array}{cccc}
G_{1} & 0 & 0 & 0 \\
0 & 0 & 0 & 0 \\
0 & 0 & G_{2} & 0 \\
0 & 0 & 0 & G_{3}
\end{array}\right]\right]\right]
$$

It should be noted that the brackets representing the VM joining nodes 1,3 , the norator joining nodes 3,2 , and the norator joining nodes 3,1 , realize a DOICCII-. The nullator between nodes 2, 4 and the norator between nodes 4,1 realizes CCII- as shown in Figure 7(c). 


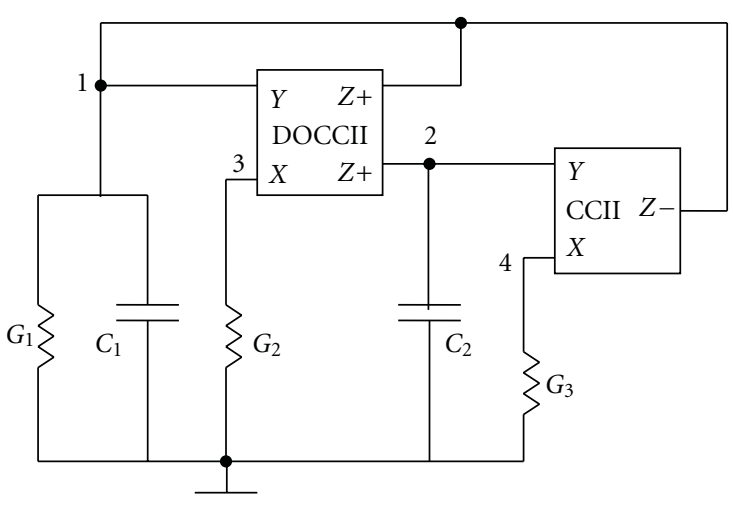

(a)

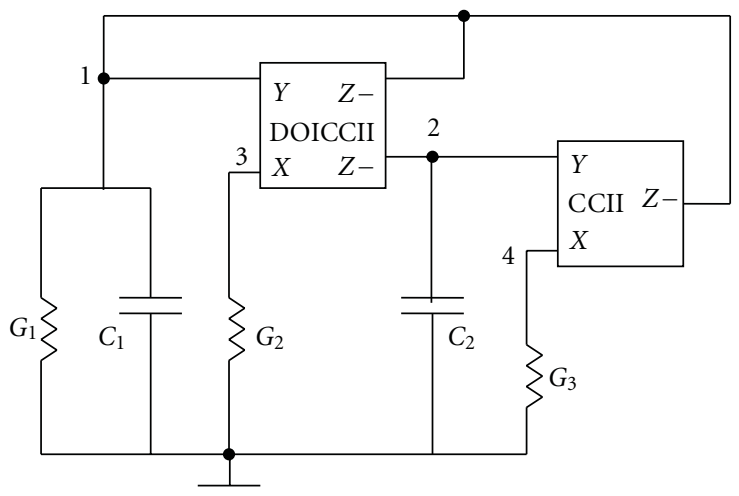

(c)

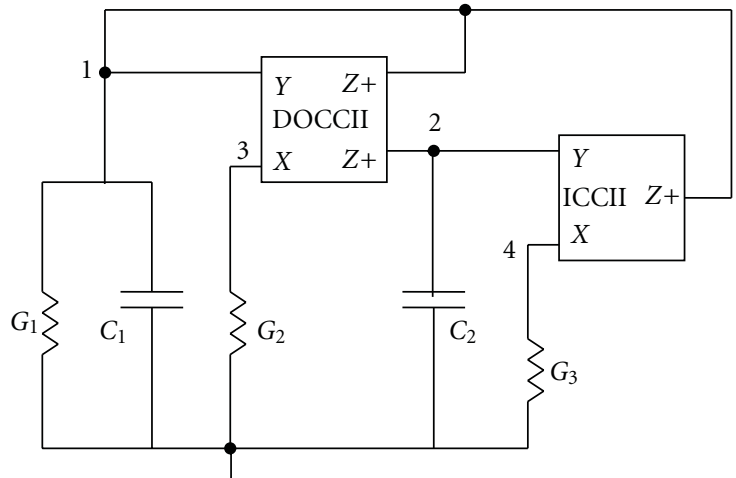

(b)

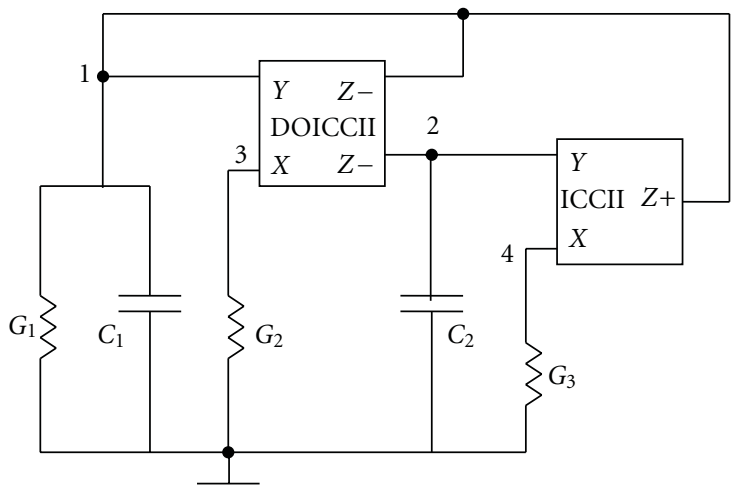

(d)

FIgURE 7: (a) Realization I of class II type B oscillator [17]. (b) Realization II of class II type-B oscillator. (c) Realization III of class II type-B oscillator. (d) Realization IV of class II type-B oscillator.

5.2.4. Realization IV. Starting from (28) and adding two blank rows and columns then following successive NAM expansion steps, the following $Y$ matrix is obtained:

$$
\left.\left.Y=\left[\begin{array}{cccc}
G_{1} & 0 & 0 & 0 \\
0 & 0 & 0 & 0 \\
0 & 0 & G_{2} & 0 \\
0 & 0 & 0 & G_{3}
\end{array}\right]\right]\right] .
$$

It should be noted that the brackets representing the VM joining nodes 1,3 , the norator joining nodes 3,2 , and the norator joining nodes 3,1 realize a DOICCII-. The VM between nodes 2, 4 and the CM between nodes 4,1 realize an ICCII+ as shown in Figure 7(d).

\section{Realization of Class II Oscillators Using Infinity Parameters}

The class II circuits generated in the previous section can also be obtained using combination of the bracket method and infinity parameters method as demonstrated in this section.

6.1. Class II-Type A. The circuit shown in Figure 6 can also be obtained using the infinity parameters describing the NAM of the BOCCII as explained next.
The NAM representation of the BOCCII is given by [11, 21]

$$
\begin{gathered}
X \\
Z- \\
Z+ \\
Z+
\end{gathered}\left[\begin{array}{cc}
\infty_{i} & -\infty_{i} \\
-\infty_{i} & \infty_{i} \\
\infty_{i} & -\infty_{i}
\end{array}\right] .
$$

From (25), adding and subtracting infinity parameter terms to the positions 1, 1 and 2, 1 as follows:

$$
\left.Y=\left[\begin{array}{cccc}
G_{1}-G_{2}-\infty_{1}+\frac{\infty_{1}^{2}}{\infty_{1}} & 0 & 0 & 0 \\
G_{2}+\infty_{1}-\frac{\infty_{1}^{2}}{\infty_{1}} & 0 & 0 & 0 \\
0 & 0 & 0 & 0 \\
0 & 0 & 0 & G_{3}
\end{array}\right]\right]
$$

Apply pivotal expansion to the third term in the 1,1 position to move $-G_{2}$ from 1,1 position to the diagonal position 3,3 as follows:

$$
\left.Y=\left[\begin{array}{cccc}
G_{1}-\infty_{1} & 0 & \infty_{1} & 0 \\
G_{2}+\infty_{1}-\frac{\infty_{1}^{2}}{\infty_{1}} & 0 & 0 & 0 \\
-\infty 1 & 0 & G_{2}+\infty_{1} & 0 \\
0 & 0 & 0 & G_{3}
\end{array}\right]\right]
$$


Next, apply also pivotal expansion to the third term in the 2, 1 position to move $G_{2}$ from 2, 1 position to the diagonal position 3, 3 as follows:

$$
\left.Y=\left[\begin{array}{cccc}
G_{1}-\infty_{1} & 0 & \infty_{1} & 0 \\
\infty_{1} & 0 & -\infty_{1} & 0 \\
-2 \infty_{1} & 0 & 2 \infty_{1}+2 G_{2} & 0 \\
0 & 0 & 0 & G_{3}
\end{array}\right]\right] .
$$

Applying the scaling of row rules demonstrated in [2] to row three and with a scaling factor of one half it follows that:

$$
\left.Y=\left[\begin{array}{cccc}
G_{1}-\infty_{1} & 0 & \infty_{1} & 0 \\
\infty_{1} & 0 & -\infty_{1} & 0 \\
-\infty_{1} & 0 & \infty_{1}+G_{2} & 0 \\
0 & 0 & 0 & G_{3}
\end{array}\right]\right] .
$$

The brackets realize a CCII+; comparing with (33) it is seen that the infinity parameters realize a BOCCII as shown in Figure 6.

The additional three equivalent class II-type-A oscillator circuits can be generated in a similar way using infinity parameters to realize the first building block and are not included here to limit paper length.

\subsection{Class II Type-B}

6.2.1. Realization I. The circuit shown in Figure 7(a) can also be obtained using the infinity parameters describing the NAM of the DOCCII as explained next.

The NAM representation of the DOCCII++ is given by

$$
\begin{array}{cc}
X & Y \\
X+ \\
Z+
\end{array}\left[\begin{array}{cc}
\infty_{i} & -\infty_{i} \\
\infty_{i} & -\infty_{i} \\
\infty_{i} & -\infty_{i}
\end{array}\right]
$$

After adding two blank rows and columns to (28), brackets are used to represent the nullator between nodes 2 and 4 and the norator between nodes 1 and 4 that are combined to move $G_{3}$ from 1, 2 position to the diagonal position 4,4 . The infinity parameters are used next to move both of $-G_{2}$ from 1,1 position and $-G_{2}$ from 2, 1 position to the diagonal position 3, 3 as follows:

$$
\left.Y=\left[\begin{array}{cccc}
-\infty_{1}+G_{1} & 0 & \infty_{1} & 0 \\
-\infty_{1} & 0 & \infty_{1} & 0 \\
-\infty_{1} & 0 & \infty_{1}+G_{2} & 0 \\
0 & 0 & 0 & G_{3}
\end{array}\right]\right] .
$$

The brackets are realizing the CCII- and the infinity parameters are realizing the DOCCII++. The above equation is realized as shown in Figure 7(a).
6.2.2. Realization II. The circuit shown in Figure 7(b) can also be obtained using the infinity parameters describing the NAM of the DOCCII as explained next.

After adding two blank rows and columns to (28), brackets are used to represent the VM between nodes 2 and 4 and the CM between nodes 1 and 4 that are combined to move $G_{3}$ from 1,2 position to the diagonal position 4,4 . The infinity parameters are used next to move both of $-G_{2}$ from 1,1 position and $-G_{2}$ from 2,1 position to the diagonal position 3, 3 as follows:

$$
\left.Y=\left[\begin{array}{cccc}
-\infty_{1}+G_{1} & 0 & \infty_{1} & 0 \\
-\infty_{1} & 0 & \infty_{1} & 0 \\
-\infty_{1} & 0 & \infty_{1}+G_{2} & 0 \\
0 & 0 & 0 & G_{3}
\end{array}\right]\right] .
$$

The brackets are realizing the ICCII+ and the infinity parameters are realizing the DOCCII++. The above equation is realized as shown in Figure 7(b).

\section{Adjoint of Class II-Oscillators}

7.1. Adjoint of Class II Type-A. Consider the NAM equation of the adjoint of the class II-type-A defined as class II-Adtype-A oscillators given by

$$
Y=\left[\begin{array}{cc}
G_{1}-G_{2} & G_{2} \\
-G_{3} & 0
\end{array}\right] .
$$

The NAM expansion is carried out using both the brackets method and the infinity parameters. The active building block to be used here is the DVCC $[13,14]$. The NAM representation of the DVCC - is given by $[11,21]$

$$
\begin{gathered}
X \\
Z-
\end{gathered}\left[\begin{array}{ccc}
X & Y_{1} & Y_{2} \\
\infty_{i} & -\infty_{i} & \infty_{i} \\
-\infty_{i} & \infty_{i} & -\infty_{i}
\end{array}\right]
$$

Add two blank rows and columns to (41) a VM between nodes 1 and 4 and a norator between nodes 2 and 4 are combined together to move $-G_{3}$ from the position 2,1 to become $G_{3}$ in the diagonal position 4,4 .

$$
\left.Y=\left[\begin{array}{cccc}
G_{1}-G_{2} & G_{2} & 0 & 0 \\
0 & 0 & 0 & 0 \\
0 & 0 & 0 & 0 \\
0 & 0 & 0 & G_{3}
\end{array}\right]\right]
$$

The infinity parameters are used next to modify the above NAM equation by moving both of $-G_{2}$ from 1,1 position and $G_{2}$ from 1, 2 to the diagonal position 3, 3, thus;

$$
Y=\left[\begin{array}{cccc}
-\infty_{1}+G_{1} & \infty_{1} & -\infty_{1} & 0 \\
0 & 0 & 0 & 0 \\
\infty_{1} & -\infty_{1} & \infty_{1}+G_{2} & 0 \\
0 & 0 & 0 & G_{3}
\end{array}\right]
$$




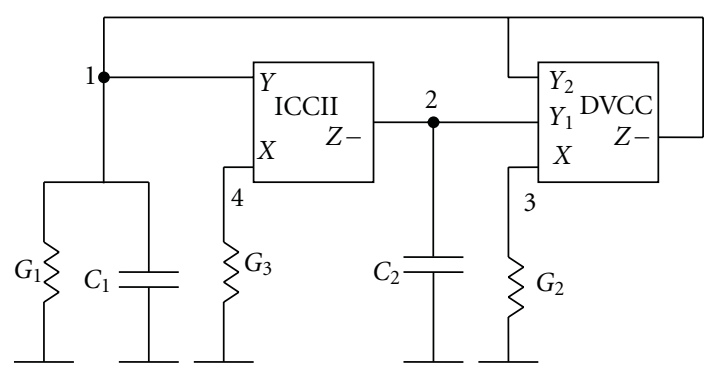

(a)

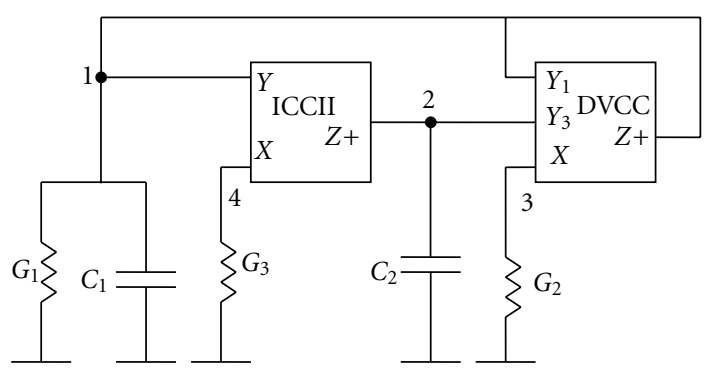

(b)

FIgURE 8: (a) Class II-Ad oscillator circuit realizing (44). (b) Class II-Ad oscillator circuit realizing (48).

Figure 8 (a) realizes the above equation using ICCII- and DVCC-. This is the adjoint circuit to that shown in Figure 6.

7.2. Adjoint of Class II Type-B. Consider the NAM equation of the adjoint of the class II type-B defined as class II-Adtype-B oscillators given by

$$
Y=\left[\begin{array}{cc}
G_{1}-G_{2} & -G_{2} \\
G_{3} & 0
\end{array}\right] .
$$

The active building block to be used here is the DVCC+ [13, 14].

The NAM representation of the DVCC+ with two noninverting $Y$ inputs $\left(Y_{1}\right.$ and $\left.Y_{3}\right)$ is given by:

$$
\underset{Z+}{X}\left[\begin{array}{ccc}
X & Y_{1} & Y_{3} \\
\infty_{i} & -\infty_{i} & -\infty_{i} \\
\infty_{i} & -\infty_{i} & -\infty_{i}
\end{array}\right] .
$$

Adding two blank rows and columns to (45); connecting a VM between nodes 1 and 4 and a CM between nodes 2 and 4 to move $\mathrm{G}_{3}$ to the diagonal position 3, 3 as follows:

$$
\left.Y=\left[\begin{array}{cccc}
G_{1}-G_{2} & -G_{2} & 0 & 0 \\
0 & 0 & 0 & 0 \\
0 & 0 & 0 & 0 \\
0 & 0 & 0 & G_{3}
\end{array}\right]\right]
$$

The infinity parameters modify the above NAM to be:

$$
\left.Y=\left[\begin{array}{cccc}
-\infty_{1}+G_{1} & -\infty_{1} & \infty_{1} & 0 \\
0 & 0 & 0 & 0 \\
-\infty_{1} & -\infty_{1} & \infty_{1}+G_{2} & 0 \\
0 & 0 & 0 & G_{3}
\end{array}\right]\right]
$$

The above equation is realized by an ICCII+ and a DVCC+ as shown in Figure 8(b).
TABLE 2: Dimensions of the MOS transistors of the circuit of Figure 9.

\begin{tabular}{cc}
\hline NMOS transistors & $\mathrm{W}(\mu \mathrm{m}) / \mathrm{L}(\mu \mathrm{m})$ \\
$M_{1}, M_{2}, M_{3}$, and $M_{4}$ & $2.5 / 1$ \\
$M_{5}$ and $M_{6}$ & $8 / 1$ \\
$M_{12}, M_{13}, M_{14}, M_{15}$, and $M_{16}$ & $20 / 2.5$ \\
\hline PMOS transistors & $\mathrm{W}(\mu \mathrm{m}) / \mathrm{L}(\mu \mathrm{m})$ \\
$M_{7}$ and $M_{8}$ & $10 / 1$ \\
$M_{9}, M_{10}, M_{11}, M_{17}$, and $M_{18}$ & $40 / 2$ \\
\hline
\end{tabular}

\section{Simulation Results}

The active building block used in all simulations included in this paper is the DVCC [13]. The DVCC is defined as a fiveport building block with a describing matrix of the form

$$
\left[\begin{array}{c}
V_{X} \\
I_{Y 1} \\
I_{Y 2} \\
I_{Z+} \\
I_{Z-}
\end{array}\right]=\left[\begin{array}{ccccc}
0 & 1 & -1 & 0 & 0 \\
0 & 0 & 0 & 0 & 0 \\
0 & 0 & 0 & 0 & 0 \\
1 & 0 & 0 & 0 & 0 \\
-1 & 0 & 0 & 0 & 0
\end{array}\right]\left[\begin{array}{c}
I_{X} \\
V_{Y 1} \\
V_{Y 2} \\
V_{Z+} \\
V_{Z-}
\end{array}\right] .
$$

The DVCC is a very powerful building block as it realizes each of CCII+, CCII-, ICCII+ and ICCII- as special cases.

Figure 9 represents the CMOS DVCC circuit [13], and the transistor aspect ratios are given in Table 2 based on the $0.5 \mu \mathrm{m}$ CMOS model from MOSIS. The supply voltages used are $\pm 1.5 \mathrm{~V}$ and $V_{\mathrm{B} 1}=-0.52 \mathrm{~V}$ and $V_{\mathrm{B} 2}=0.33 \mathrm{~V}$.

Figure $10(\mathrm{a})$ represents the output voltage waveform of the oscillator of Figure 4(b) designed for oscillation frequency equal to $1 \mathrm{MHz}$ by taking $C_{1}=C_{2}=40 \mathrm{pF}$, $R_{1}=R_{2}=R_{3}=R_{4}=4 \mathrm{k} \Omega$. To start oscillations $R_{1}$ is increased to $4.2 \mathrm{k} \Omega$. It should be noted that the simulations given are based on the above values of circuit components with no compensation. The simulation results indicate an oscillation frequency slightly lower than $1 \mathrm{MHz}$ due to the addition of the parasitic elements $R_{X 1}$ added to $R_{2}, R_{X 2}$ is added to $R_{3}$, and $R_{X 3}$ added to $R_{4}$. The parasitic capacitances are also affecting the oscillation frequency since $C_{Z 1}$ is added to $C_{1}$ and $C_{Z 2}$ is added to $C_{2}$. The total power dissipation is given by $2.8731 \mathrm{~mW}$. 


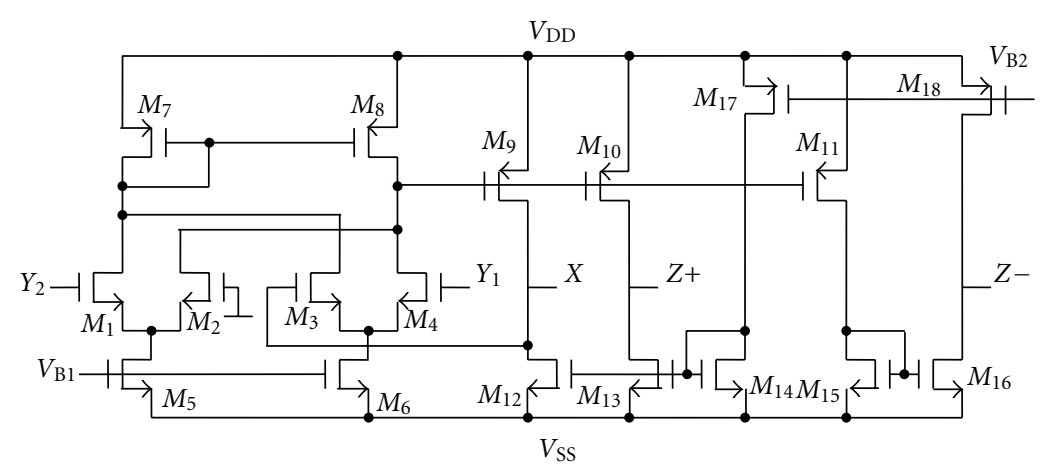

FIGURE 9: CMOS circuit of the DVCC [13].



(a)



(b)



(c)

Figure 10: (a) Simulated output waveform of the circuit of Figure 4(b). (b) Simulated output waveform of the circuit of Figure 5(b). (c) Simulated output waveform of the circuit of Figure 8(a). 
Figure 10(b) represents the output voltage waveform of the oscillator of Figure 5(b) designed for oscillation frequency equal to $1 \mathrm{MHz}$ by taking the same design values as above. The circuit operates well with the design value of $R_{1}$ without increasing its value as in the circuit of Figure 4(b). The total power dissipation is given by $2.92668 \mathrm{~mW}$.

Figure 10(c) represents the output voltage waveform of the oscillator of Figure 8(a) designed for oscillation frequency equal to $1 \mathrm{MHz}$ by taking $C_{1}=C_{2}=40 \mathrm{pF}$, $R_{1}=R_{2}=R_{3}=4 \mathrm{k} \Omega$. To start oscillations $R_{1}$ is increased to $4.4 \mathrm{k} \Omega$. The total power dissipation is given by $1.90799 \mathrm{~mW}$.

\section{Conclusions}

A new approach in the systematic synthesis of grounded passive elements canonic oscillators is given. The synthesis procedure is based on the generalized systematic synthesis framework using NAM expansion and infinity parameters. The suggested use of the NAM expansion method in the synthesis of oscillator circuits provided many new oscillator circuits to complete the families of known oscillator circuits. The active building blocks that have been considered are the CCII, ICCII, BOCCII, DOCCII, BOICCII and DOICCII. This is the first paper in the literature which uses infinity parameters in the synthesis of oscillator circuits. The oscillators generated in this paper enjoy the advantages of having independent control on the condition of oscillation by varying $R_{1}$ and on the frequency of oscillation by varying $R_{3}$. The oscillator circuits that belong to the topology of the class I generated in this paper and to class II have the advantage of being easily compensated for the parasitic effects of the current conveyors. The adjoint to class I oscillators is also considered, and it is found that the class I oscillator is selfadjoint. The adjoint of the class II oscillators using the DVCC as the basic building block is also given. Simulation results demonstrating the practicality of the circuits are included. The advantage of using the nullators, norators, VM and $\mathrm{CM}$ in symbolic circuit analysis has been most recently demonstrated in the literature [22]. It is worth noting that the generic algorithms given in [23] can also be extended to different types of pathological elements.

\section{Acknowledgment}

The author thanks the reviewers for their useful comments.

\section{References}

[1] D. G. Haigh, T. J. W. Clarke, and P. M. Radmore, "Symbolic framework for linear active circuits based on port equivalence using limit variables," IEEE Transactions on Circuits and Systems I, vol. 53, no. 9, pp. 2011-2024, 2006.

[2] D. G. Haigh, F. Q. Tan, and C. Papavassiliou, "Systematic synthesis of active-RC circuit building-blocks," Analog Integrated Circuits and Signal Processing, vol. 43, no. 3, pp. 297-315, 2005.

[3] D. G. Haigh and P. M. Radmore, "Admittance matrix models for the nullor using limit variables and their application to circuit design," IEEE Transactions on Circuits and Systems I, vol. 53, no. 10, pp. 2214-2223, 2006.
[4] D. G. Haigh, "A method of transformation from symbolic transfer function to active-RC circuit by admittance matrix expansion," IEEE Transactions on Circuits and Systems I, vol. 53, no. 12, pp. 2715-2728, 2006.

[5] H. J. Carlin, "Singular network elements," IEEE Transactions on Circuit Theory, vol. 11, pp. 67-72, 1964.

[6] I. A. Awad and A. M. Soliman, "Inverting second generation current conveyors: the missing building blocks, CMOS realizations and applications," International Journal of Electronics, vol. 86, no. 4, pp. 413-432, 1999.

[7] I. A. Awad and A. M. Soliman, "On the voltage mirrors and the current mirrors," Analog Integrated Circuits and Signal Processing, vol. 32, no. 1, pp. 79-81, 2002.

[8] I. A. Awad and A. M. Soliman, "A new approach to obtain alternative active building blocks realizations based on their ideal representations," Frequenz, vol. 54, no. 11-12, pp. 290299, 2000.

[9] R. A. Saad and A. M. Soliman, "Generation, modeling, and analysis of CCII-based gyrators using the generalized symbolic framework for linear active circuits," International Journal of Circuit Theory and Applications, vol. 36, no. 3, pp. 289-309, 2008.

[10] R. A. Saad and A. M. Soliman, "Use of mirror elements in the active device synthesis by admittance matrix expansion," IEEE Transactions on Circuits and Systems I, vol. 55, no. 9, pp. 27262735, 2008.

[11] R. A. Saad and A. M. Soliman, "On the systematic synthesis of CCII-based floating simulators," International Journal of Circuit Theory and Applications, vol. 38, no. 9, pp. 935-967, 2010.

[12] A. S. Sedra and K. C. Smith, "A second generation current conveyor and its applications," IEEE Transactions on Circuit Theory, vol. 132, pp. 132-134, 1970.

[13] H. O. Elwan and A. M. Soliman, "Novel CMOS differential voltage current conveyor and its applications," IEE Proceedings, vol. 144, no. 3, pp. 195-200, 1997.

[14] W. Chiu, S. I. Liu, H. W. Tsao, and J. J. Chen, "CMOS differential difference current conveyors and their applications," IEE Proceedings, vol. 143, no. 2, pp. 91-96, 1996.

[15] A. M. Soliman, "Simple sinusoidal active RC oscillators," International Journal of Electronics, vol. 39, no. 4, pp. 455-458, 1975.

[16] A. M. Soliman, "Synthesis of grounded capacitor and grounded resistor oscillators," Journal of the Franklin Institute, vol. 336, no. 4, pp. 735-746, 1999.

[17] A. M. Soliman, "Current mode CCII oscillators using grounded capacitors and resistors," International Journal of Circuit Theory and Applications, vol. 26, no. 5, pp. 431-438, 1998.

[18] B. B. Bhattacharyya and M. N. S. Swamy, "Network transposition and its application in synthesis," IEEE Transactions on Circuit Theory, vol. 18, pp. 394-397, 1971.

[19] A. M. Soliman, "Adjoint network theorem and floating elements in the NAM," Journal of Circuits, Systems and Computers, vol. 18, no. 3, pp. 597-616, 2009.

[20] A. M. Soliman, "Generation of current conveyor based oscillators using nodal admittance matrix expansion," Analog Integrated Circuits and Signal Processing, vol. 65, no. 1, pp. 43$59,2010$.

[21] A. M. Soliman, "On the DVCC and the BOICCII as adjoint elements," Journal of Circuits, Systems and Computers, vol. 18, no. 6, pp. 1017-1032, 2009. 
[22] E. Tlelo-Cuautle, M. A. Duarte-Villaseñor, and I. GuerraGómez, "Automatic synthesis of VFs and VMs by applying genetic algorithms," Circuits, Systems, and Signal Processing, vol. 27, no. 3, pp. 391-403, 2008.

[23] C. Sánchez-López, F. V. Fernández, E. Tlelo-Cuautle, and S. X. D. Tan, "Pathological element-based active device models and their application to symbolic analysis," IEEE Transactions on Circuits and Systems I, vol. 58, no. 6, pp. 1382-1395, 2011. 



Submit your manuscripts at

http://www.hindawi.com
